\title{
Chromone-3-carboxylic acid as a potential electron scavenger: a surface-enhanced Raman scattering study
}

\author{
N. F. L. Machado, ${ }^{a}$ C. Ruano, ${ }^{b}$ J. L. Castro, ${ }^{b}$ M. P. M. Marques ${ }^{a}$ and \\ J. C. Otero ${ }^{* b}$
}

Received 14th July 2010, Accepted 1st October 2010

DOI: $10.1039 / \mathrm{c0cp01174f}$

A SERS study of chromone 3-carboxylic acid adsorbed on silver colloids was undertaken, in order to assess the ability of this compound to accept electrons in charge transfer (CT) processes. Theoretical SERS intensities under photoinduced CT resonant conditions have been carried out for both the neutral and the deprotonated species allowing to conclude,

by comparison with the experimental data, that the recorded SER corresponds to the anionic form of the acid linked to the metal. It was shown that the SERS-CT mechanism predominates for this particular compound, thus explaining the strong enhancement of the band at $c a$. $1600 \mathrm{~cm}^{-1}$ assigned to the $8 \mathrm{a}$ ring stretching mode. The identification of CT processes is of the utmost importance for understanding the mechanism through which these benzopyranes may act as antioxidants.

\section{Introduction}

Benzopyrane-type heterocyclic compounds are naturally occurring compounds ubiquitously distributed in plants. Chromones (1-benzopyran-4-ones) and chromone derivatives in particular are present in representative amounts in a normal human diet and possess a wide spectrum of biological activities such as antifungal, antimicrobial, antiviral, antiallergic, anti-inflammatory and antitumour. This is mainly due to their well-recognised antioxidant properties, which stem from their ability to neutralise active forms of oxygen and to cut off free radical processes. ${ }^{1-7}$ Therefore, this group of molecules has been the object of intense research in the last decade, ${ }^{8}$ aiming at the development of promising antioxidant agents with chemopreventive capacity (mainly against neoplastic and neurodegenerative disorders). Actually, in view of their high stability and low toxicity, this kind of compounds may constitute a safe and reliable option for use as additives in food and pharmaceutical products, since a great number of the antioxidants currently employed are being rejected due to their deleterious toxic effects in humans. ${ }^{9}$

Therefore, the study of the mechanisms through which these compounds act, both in vitro and in vivo, is of the utmost relevance for understanding their pertinent biological role, allowing a rational design of novel and more efficient antioxidants for use as anti-inflammatory and hopefully anticancer chemopreventive agents. Since there is a close relationship between activity and structure, ${ }^{10}$ it is of paramount importance to disclose the structural and conformational features that are related with a possible antioxidant activity.

\footnotetext{
"Unidade I\&D "Quimica-Física Molecular",

Faculdade de Ciências e Tecnologia, Universidade de Coimbra,

3000 Coimbra, Portugal. E-mail: jc_otero@uma.es;

Fax: + 34 952132019; Tel: + 34952132019

${ }^{b}$ Departamento de Quimica Física, Facultad de Ciencias,

Universidad de Málaga, Unidad Asociada CSIC, E-29071 Málaga,

Spain
}

The Surface Enhanced Raman Scattering (SERS) is a complex phenomenon capable of producing an enormous enhancement of the Raman signal of some molecules adsorbed on nanostructured surfaces of alkaline or coinage metals, especially silver. Generally speaking, when a molecule interacts with such metals, vibrational frequency shifts and changes between the relative intensities of the Raman and SERS bands can be seen. From the analysis of these data it is possible to extract information concerning the adsorption of the molecule and the nature of the SERS enhancement mechanism. Most of the reported SERS studies of aromatic molecules ${ }^{11,12}$ have been interpreted in the light of the so called electromagnetic enhancement mechanism (EM), which would allow to determine the orientation of the adsorbate relative to the metal surface. Therefore, The EM selection rules of SERS ${ }^{11}$ relate the perpendicular or parallel orientation of planar molecules with respect to the surface, with a consequent enhancement of the in-plane or out-of-plane normal modes, respectively. However, although this enhancement mechanism is nowadays recognised as general in SERS, it does not account for the selective enhancement of bands with the same symmetry which, in turn, could be explained by the presence of charge transfer (CT) enhancement processes. These are strongly dependent on the nature of the molecule, the metal and the particular experimental conditions, and can be envisaged as a resonant photoinduced electron transfer process between the metal and the molecule. Hence, the prime task when analysing a SERS is to identify the main mechanisms, EM and/or CT, involved and how much each one contributes to the observed spectra, in a particular experience. This preliminary question when analysing SERS data remains unclear and has been the object of controversy for many years.

In this concern, our research group has developed a methodology for detecting the presence of CT processes in SERS on the basis of quantum chemical calculations. ${ }^{13-15}$ Once the CT mechanism is confirmed, the SERS spectrum may provide very useful information on the electron acceptor 


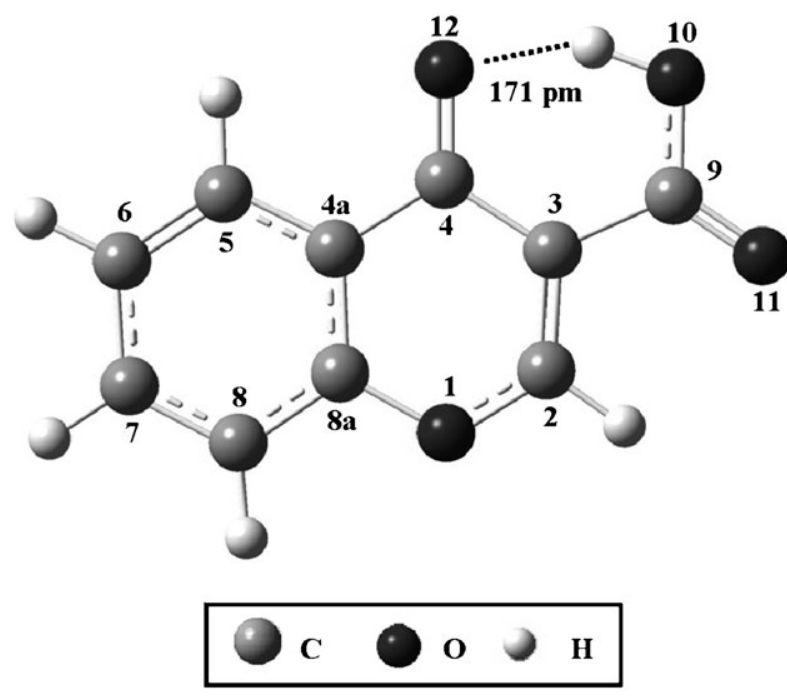

Fig. 1 B3LYP/6-31G** optimised geometry for the lowest energy conformer of chromone-3-carboxylic acid (3CA), comprising the most stable intramolecular H-bond.

properties of the adsorbate. For aromatic systems such as chromones, this is a very important issue in order to check their capabilities for use as efficient radical scavengers.

The present work reports the SERS recorded on silver sols for an acidic chromone derivative, chromone-3-carboxylic acid (3CA, Fig. 1). The experimental data were analysed in the light of the CT selection rules, by considering two possible adsorption mechanisms: through the neutral molecule or via its deprotonated anion. In order to identify the SERS active adsorbate, the theoretical SERS-CT intensities of these two species were calculated and compared with the experimental results. Moreover, a tentative vibrational assignment was proposed, based on Density Functional Theory (DFT) force field calculations and on previous experimental studies on analogous molecules.

\section{Experimental}

\section{Chemicals and colloid preparation}

Chromone-3-carboxylic acid was purchased from SigmaAldrich Quimica S.A. (Sintra, Portugal) at the single available purity $(97 \%)$. For preparing the aqueous solutions, complete solubilisation of the compound was achieved by adding $\mathrm{NaOH}-1 \mathrm{M}$ until $\mathrm{pH}=9$.

Colloidal silver solutions were prepared using triply distilled water, according to the method of Creighton et al. ${ }^{16}$ fully described elsewhere. ${ }^{17}$ This procedure basically consists in reducing an aqueous solution of $10^{-3} \mathrm{M} \mathrm{AgNO}_{3}$ with an excess of cooled $\left(0-5{ }^{\circ} \mathrm{C}\right) \mathrm{NaBH}_{4}$, at a $1: 3$ ratio of $1 \times 10^{-3} \mathrm{MAgNO}_{3}$ and $2 \times 10^{-3} \mathrm{M} \mathrm{NaBH}_{4}$, respectively. After stirring for some time to allow homogenisation, the mixture was left to rest at room temperature for $c a .90 \mathrm{~min}$. In some cases, during this waiting period a dark colour appears in the heart of the solution, which urges for vigorous stirring in order to stabilise the colloid. This should be a transparent, yellow solution, displaying an absorption maximum at $390 \mathrm{~nm}$. The adsorbate (tested compound) is then added to the colloid, a change in colour from the initial yellowish to a final blue-greenish being observed when it adsorbs to the colloid particles and induces their aggregation.

\section{Raman spectroscopy}

The Raman spectra of both the solid sample and the saturated aqueous solution $(\mathrm{pH}=9)$ were obtained at room temperature, in a triple monochromator Jobin-Yvon T64000 Raman system (focal distance $0.640 \mathrm{~m}$, aperture $f / 7.5$ ), with holographic gratings of 1800 grooves $\mathrm{mm}^{-1}$. The premonochromator stage was used in the subtractive mode. The detection system was a liquid nitrogen cooled nonintensified $1024 \times 256$ pixel $\left(1^{\prime \prime}\right)$ Charge Coupled Device (CCD). The entrance slit was set to $200 \mu \mathrm{m}$, and the slit between the premonochromator and the spectrograph was opened to $12 \mathrm{~mm}$.

The excitation radiation was provided $(c a .100 \mathrm{~mW}$ at the sample position) by the $514.5 \mathrm{~nm}$ line of an $\mathrm{Ar}^{+}$laser (Coherent, model Innova 300). A $90^{\circ}$ geometry between the incident radiation and the collecting system was employed. Under the above mentioned conditions, the error in measured wavenumbers was estimated to be within $1 \mathrm{~cm}^{-1}$. The samples were sealed in Kimax glass capillary tubes of $0.8 \mathrm{~mm}$ inner diameter.

The SERS spectra were recorded in a Jobin-Yvon U-1000 double monochromator spectrometer fitted with a cooled Hamamatsu R943-02 photomultiplier, using the $514.5 \mathrm{~nm}$ exciting line from a Spectra-Physics $2020 \mathrm{Ar}^{+}$laser $(c a .50 \mathrm{~mW}$ at the sample). The $200 \mu \mathrm{m}$ slit width was kept constant, allowing a spectral resolution of $4 \mathrm{~cm}^{-1}$. The wavenumbers were measured considering the same laser plasma lines as standards, whereby a precision of $c a .2 \mathrm{~cm}^{-1}$ was obtained. A $10 \mathrm{~mm}$ width quartz cell was used as the sample container.

\section{DFT calculations}

The quantum mechanical calculations were performed using the GAUSSIAN 03W program, ${ }^{18}$ within DFT approach, in order to properly account for the electron correlation effects which are particularly important in this kind of conjugated systems. The widely employed hybrid method denoted by B3LYP, which includes a mixture of HF and DFT exchange terms and the gradient-corrected correlation functional of Lee, Yang and Parr ${ }^{19,20}$ as proposed and parametrised by Becke $^{21,22}$ was used, along with the double-zeta split valence basis set $6-31 \mathrm{G}^{* *} .{ }^{23}$ Molecular geometries were fully optimised by the Berny algorithm, using redundant internal coordinates. ${ }^{24}$ The bond lengths to within $c a .0 .1 \mathrm{pm}$ and the bond angles to within $c a .0 .1^{\circ}$. The final root-mean-square (rms) gradients were always less than $3 \times 10^{-4}$ hartree bohr $^{-1}$ or hartree radian $^{-1}$.

Calculation of the harmonic vibrational wavenumbers was carried out at the same level of theory for the most stable conformer of 3CA (Fig. 1), both for the neutral acid and its carboxylate anion, in order to obtain the theoretical vibrational spectra (Raman and infrared). The geometries of the optimised planar $\mathrm{C}_{\mathrm{s}}$ structures for both species were kept constant when calculating the properties of the respective radicals. 


\section{Results and discussion}

\section{Detecting CT processes in SERS}

Our research group has developed a methodology for detecting the presence of the CT mechanism in a particular SERS experience. ${ }^{13-15}$ In essence, this is based on the assumption that the SERS-CT enhancement mechanism is analogous to a Resonance Raman (RR) process, ${ }^{25}$ where the incident photon $(h \nu)$ leads to a resonant transfer of one electron from the Fermi level of the metal to vacant orbitals of the adsorbate, yielding the corresponding transient radical. This SERS-CT mechanism implies the photoinduced electron transfer from the metal $(\mathrm{M})$ to the adsorbate $(\mathrm{A})$ :

$$
\mathrm{M}-\mathrm{A}+h \nu \rightarrow \mathrm{M}^{+}-\mathrm{A}^{\bullet-}
$$

Therefore, the transient excited state of the surface complex $\mathrm{M}^{+}-\mathrm{A}^{\bullet-}$ shows $\mathrm{CT}$ character. In the reverse process, the system returns to the initial state emitting a Raman photon $\left(h \nu^{\prime}\right)$ if the molecule remains vibrationally excited $\left(\mathrm{A}^{*}\right)$ :

$$
\mathrm{M}^{+}-\mathrm{A}^{\bullet-} \rightarrow \mathrm{M}-\mathrm{A}^{*}+h \nu^{\prime}
$$

An important difference exists between neutral adsorbates as pyridine (A) and organic carboxylic acids, given that the latter can be adsorbed as carboxylates $\left(\mathrm{A}^{-}\right)$which are already charged in the ground state. In this case, the respective dianion radical $\left(\mathrm{A}^{\cdot 2-}\right)$ will be formed in the $\mathrm{CT}$ excited state, a much less stable species since it supports two negative charges:

$$
\mathrm{M}-\mathrm{A}^{-}+h \nu \rightarrow \mathbf{M}^{+}-\mathbf{A}^{\cdot 2-}
$$

and

$$
\mathrm{M}^{+}-\mathrm{A}^{\cdot 2-} \rightarrow \mathrm{M}-\mathrm{A}^{-*}+h \nu^{\prime}
$$

Therefore, there are two different possibilities for the SERS-CT mechanism, depending on the adsorption of either the neutral or the anionic species. This mechanism sets the resonance between the ground (singlet ground state $\mathrm{S}_{0}$ : $\mathrm{M}-\mathrm{A}$ or $\mathrm{M}-\mathrm{A}^{-}$) and excited $\mathrm{CT}$ states of the surface complex (singlet excited charge transfer states $\mathrm{CT}_{\mathrm{i}}: \mathrm{M}^{+}{ }_{-} \mathrm{A}^{\bullet-}$ or $\mathrm{M}^{+}-\mathrm{A}^{\cdot 2-}$ ). A reasonable approach is to consider only the states of the adsorbate in these transitions. Under this simpler view, the resonance would involve the corresponding species, either neutral or anionic, in their singlet ground states (A or $\left.\mathrm{A}^{-} ; \mathrm{S}_{0}\right)$ and their radial anion, or dianion, in their respective doublet ground states $\left(\mathrm{A}^{\bullet-}\right.$ or $\left.\mathrm{A}^{\bullet 2-} ; \mathrm{D}_{0}\right)$.

It is generally accepted that the A-term is the most important contribution in an RR process. This term becomes non-zero when the dipole transition moments associated with the resonant electronic transition and the products of the vibrational overlap integrals (Franck-Condon factors) are non-zero. The second condition is related to differences between the equilibrium geometries of the states involved in the electronic transition (Tsuboi's rule). If the most enhanced SERS bands correspond to those vibrations connecting the geometry of the minima of the resonant states (A and $\mathrm{A}^{\bullet-}$ for the neutral, or $\mathrm{A}^{-}$and $\mathrm{A}^{\bullet 2-}$ for the carboxylate), the presence of a resonant CT process can be confirmed. The lack of data referring to molecular properties such as equilibrium structures for radicals of benzene-like molecules urges for quantum chemical calculations in order to foresee the effect of resonant CT processes in the SERS bands of this kind of systems.

Theoretical SERS-CT intensities for each molecular species $\mathrm{A}$ and $\mathrm{A}^{-}$have been estimated following the equations of Wolde et $a l .{ }^{26}$ for an RR process. According to these authors, the relative intensity of a particular normal mode $j$ can be estimated by using the equation:

$$
I_{\mathrm{SERS}-\mathrm{CT}, j}=\Re B_{j}^{2} \omega_{j}^{2}
$$

where $\omega_{j}$ is the wavenumber corresponding to the $j$-normal mode in the ground electronic state; $\Re$ is a constant that can be arbitrarily adjusted to normalise the relative intensities; and $B_{j}$ is a parameter related to the difference between the equilibrium geometries of the two states involved in the RR process; this parameter can be obtained by computing the molecular forces $(f)$ at the Franck-Condon point of the potential energy surface for the excited electronic state, i.e. the respective radicals, and is given by the expression

$$
B_{j}=\left(2.41 \times 10^{6}\right) f \sqrt{M} L_{j} \omega_{j}^{-3 / 2}
$$

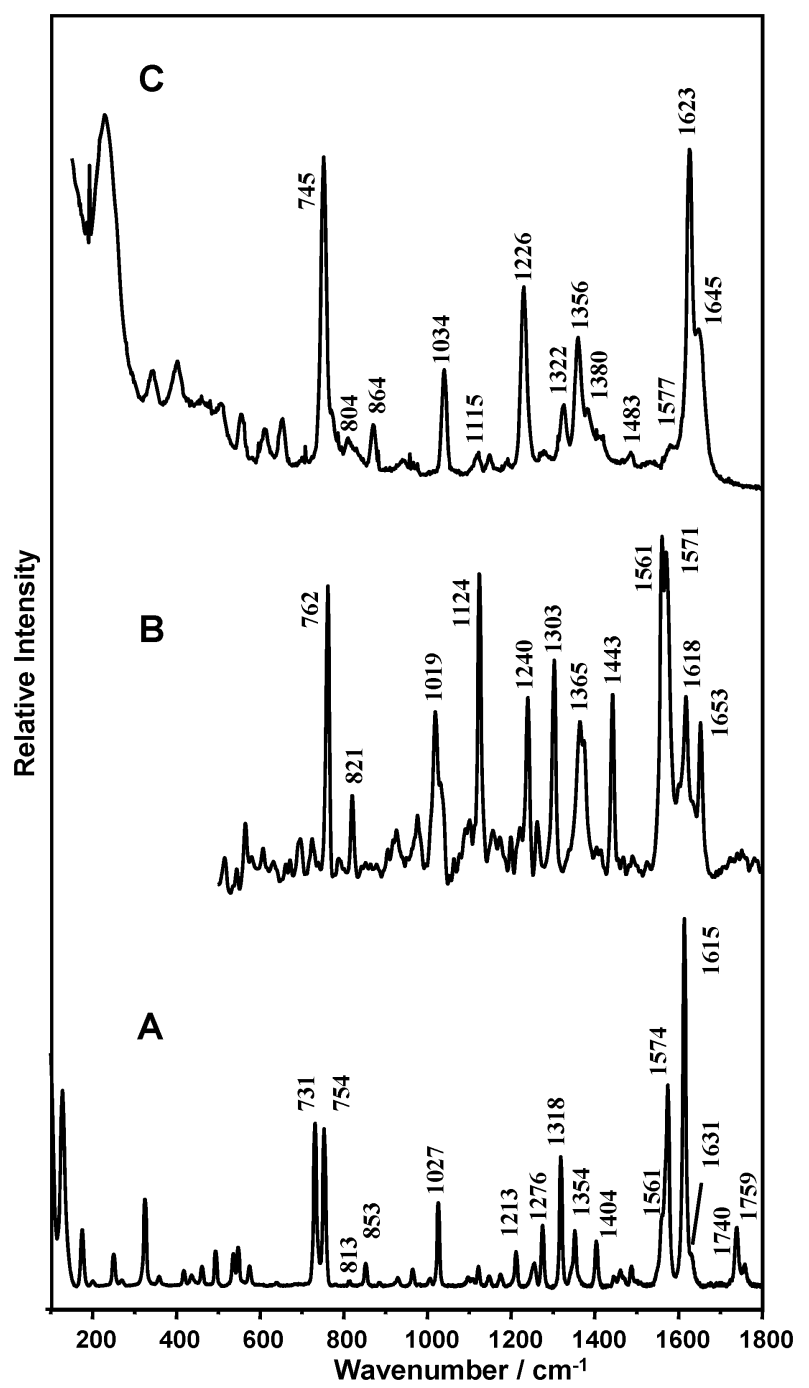

Fig. 2 Raman spectra of chromone-3-carboxylic acid (3CA) in the solid (A) and in aqueous solution at $\mathrm{pH}=9$ (B), and corresponding SERS spectrum on silver sols (C). 
$L$ representing the normal mode matrix of the ground electronic state, and $M$ the matrix of atomic masses. The resulting calculated SERS-CT spectra have been drawn by using Gaussian functions, with an area proportional to the relative intensities located at the B3LYP/6-31G** calculated wavenumbers.

This methodology enabled to explain the selective enhancements previously observed in the SERS of different aromatic molecules such as pyrimidine, ${ }^{13}$ pyridine ${ }^{27}$ or pyrazine, ${ }^{28}$ and their derivatives ${ }^{15,29,30}$ 5-fluorouracil ${ }^{31}$ or carboxylic acids. $^{32,33}$ These studies ${ }^{13-15}$ have allowed to conclude that the main feature of the SERS-CT spectra for benzene-like molecules is the strong enhancement of the band corresponding to the aromatic ring stretching mode $8 \mathrm{a},{ }^{34}$ recorded at about $1600 \mathrm{~cm}^{-1}$.

\section{Raman and SERS results}

The solubility of 3CA is very scarce in water, and it is thus necessary to increase the $\mathrm{pH}$ of the sample in order to obtain a concentration which allows the observation of bands with sufficient intensity. Fortunately, organic carboxylic acids are known to adsorb on silver as carboxylates, and therefore the SERS intensities have to be compared with those of the Raman spectrum recorded for the aqueous solution of the deprotonated species. The Raman spectra of solid 3CA and its aqueous solution at $\mathrm{pH}=9$ show very different intensities (Fig. 2A and B), due to changes in the normal vibrational modes coupled to specific intermolecular interactions. The latter include top-to-top hydrogen bonds between carboxylic groups of adjacent molecules in the solid, which disappear in alkaline aqueous solutions when $3 \mathrm{CA}$ is deprotonated at the $\mathrm{COOH}$ moiety. This leads to spectral changes in the $1550-1700 \mathrm{~cm}^{-1}$ region, where several fundamentals assigned to ring stretchings of the benzene moiety ( $8 \mathrm{a}$ and $8 \mathrm{~b}$ modes) are observed, coupled in a complex way with other coordinates involving the stretching of the conjugated bonds $\mathrm{C}_{4}=\mathrm{O}_{12}$, $\mathrm{C}_{2}=\mathrm{C}_{3}$ and the carboxylic $\mathrm{C}_{9}=\mathrm{O}_{11}$ (in the case of the acid) or the carboxylate $\mathrm{O}_{10} \mathrm{C}_{9} \mathrm{O}_{11}$ antisymmetric stretching (in the case of the anion) (Table 1).

Table 1 Experimental Raman (solid and aqueous solution $\mathrm{pH}=9$ ) and SERS wavenumbers $\left(\mathrm{cm}^{-1}\right.$ ), and $\mathrm{B} 3 \mathrm{LYP} / 6-31 \mathrm{G}^{* *}$ calculated wavenumbers for the neutral and anionic species of chromone-3-carboxylic acid (3CA)

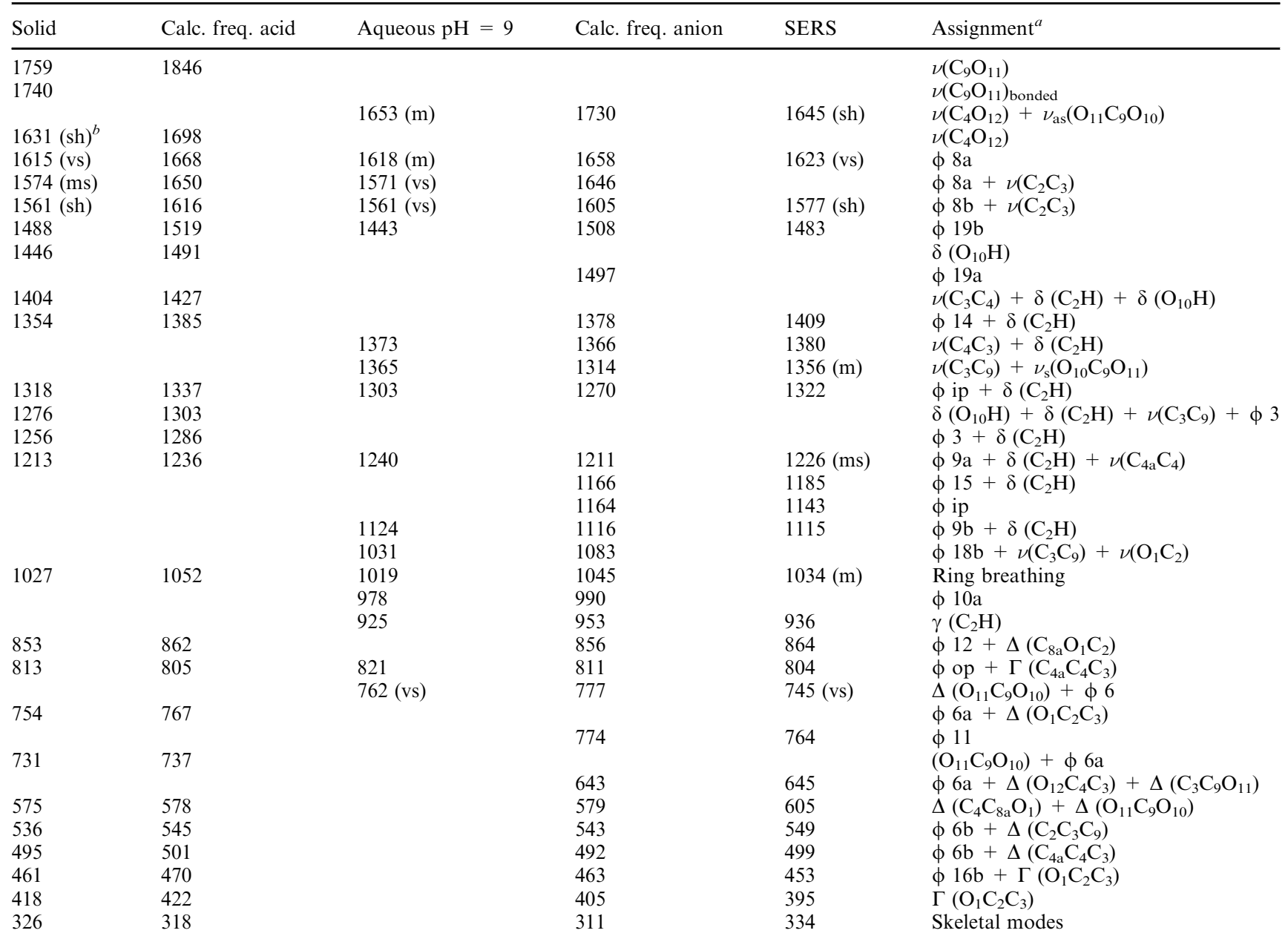

${ }^{a}$ Atoms are numbered according to Fig. 1. The Wilson notation was used for the description of benzene derivatives normal vibrations; ${ }^{34,35}$ for inplane fundamentals: $\mathrm{C}-\mathrm{C}$ stretching vibrations $(8 \mathrm{a}, 8 \mathrm{~b}, 14,19 \mathrm{a}, 19 \mathrm{~b}), \mathrm{C}-\mathrm{H} / \mathrm{X}$ bending vibrations $(3,18 \mathrm{a}, 18 \mathrm{~b})$, radial skeletal vibrations $(1,6 \mathrm{a}, 6 \mathrm{~b}$, 12) C-H stretching vibrations (2, 20a, 20b, 7a, 7b); for out-of-plane fundamentals: $\mathrm{C}-\mathrm{H} / \mathrm{X}$ vibrations $(5,10 \mathrm{a}, 11,17 \mathrm{a}, 17 \mathrm{~b})$, skeletal vibrations (4, 16a, 16b). $\delta$-in-plane deformation, $\gamma$ - out-of-plane deformation, $\Delta$-in-plane deformation of skeleton atoms, $\Gamma$-out-of-plane deformation of skeleton atoms. ${ }^{b}$ Raman intensities: sh—shoulder, w—weak, m—-medium, ms - medium-strong, s—strong, vs—very strong. 
Four bands are recorded at 1561 (sh), 1574 (ms), 1615 (vs) and $1631(\mathrm{sh}) \mathrm{cm}^{-1}$ for the solid sample, which correlate with those detected at 1561 (vs), 1571 (vs), 1618 (m) and $1653(\mathrm{~m})$ $\mathrm{cm}^{-1}$ for the aqueous solution (Fig. 2) The B3LYP force field predicts four fundamentals in this region, at 1616, 1650, 1668 and $1698 \mathrm{~cm}^{-1}$ for the neutral species and at $1605,1646,1658$ and $1730 \mathrm{~cm}^{-1}$ for the carboxylate anion, respectively. These vibrational modes are represented in Fig. 3 where a good correlation between the lowest and highest wavenumber modes of both species can be appreciated. These are calculated at 1616 and $1698 \mathrm{~cm}^{-1}$ for the neutral form and at 1605 and $1730 \mathrm{~cm}^{-1}$ for the anion, in agreement with the experimental blue shift observed for the latter when 3CA loses the carboxylic proton. However a significant mode rotation between the remaining fundamentals at $c a .1650$ and $1660 \mathrm{~cm}^{-1}$ occurs, changing the amplitude, direction and phase of the atom displacements.

When comparing the Raman spectra for 3CA in aqueous solution with the corresponding SERS, the most striking result is again the intensity change of the bands recorded in the $1550-1700 \mathrm{~cm}^{-1}$ region, especially the selective enhancement of the SERS band centered at $1623 \mathrm{~cm}^{-1}$ (Fig. 2B and C). This is the strongest band in the SERS spectra and correlates well with the medium intensity line recorded at $1618 \mathrm{~cm}^{-1}$ in the aqueous solution Raman. On the contrary, the strongest Raman bands at 1561 and $1571 \mathrm{~cm}^{-1}$ show very weak intensities in SERS, while the $1653 \mathrm{~cm}^{-1}$ line appears in SERS as a shoulder at $1645 \mathrm{~cm}^{-1}$. The remaining principal SERS bands at 1356, 1226, 1034 and $745 \mathrm{~cm}^{-1}$ display lower intensities and are assigned to in-plane vibrational modes. In order to account for the selective enhancement of the $1623 \mathrm{~cm}^{-1}$ feature, it is necessary to refer to the selection rules of the CT enhancement mechanism, drawn in Fig. 3.

When the CT mechanism operates, the incoming photon produces the resonant transfer of an electron from the metal to the adsorbate, leading to the formation of the radical dianion. When this electron is located in the Lowest Unoccupied Molecular Orbital (LUMO), the bonding and antibonding interactions are responsible for the transient forces acting on the nuclei ( $f$ vector in eqn (6)). Therefore, the B3LYP computed force vector (Fig. 3) indicates the direction towards the equilibrium geometry of the transient radical. This vector contains the selection rules of the SERS-CT mechanism and determines which Raman vibrations should be enhanced, by means of eqn (5) and (6). Accordingly, the shapes of the LUMO and of the $f$ vector are closely related, and involve the stretching of $\mathrm{C}_{4}=\mathrm{O}_{12}$ and $\mathrm{O}_{10} \mathrm{C}_{9} \mathrm{O}_{11}$ conjugated bonds and benzenic CC. In the case of the anion, the normal mode calculated at $1658 \mathrm{~cm}^{-1}$, and the corresponding forces, have very similar shapes thus accounting for the selective SERS enhancement of the band at $1623 \mathrm{~cm}^{-1}$.

This qualitative analysis may be further quantified through the calculated band intensities, also pictured in Fig. 3. The theoretical $I_{\text {SERS-CT }}$ spectrum for the deprotonated 3CA predicts the selective enhancement of a single band at $1658 \mathrm{~cm}^{-1}\left(I_{\text {SERS-CT,1658 }}=100\right)$, in perfect agreement with the SERS observed experimentally (Fig. 3 and 4). In contrast, the correlation between the forces and the atomic displacement
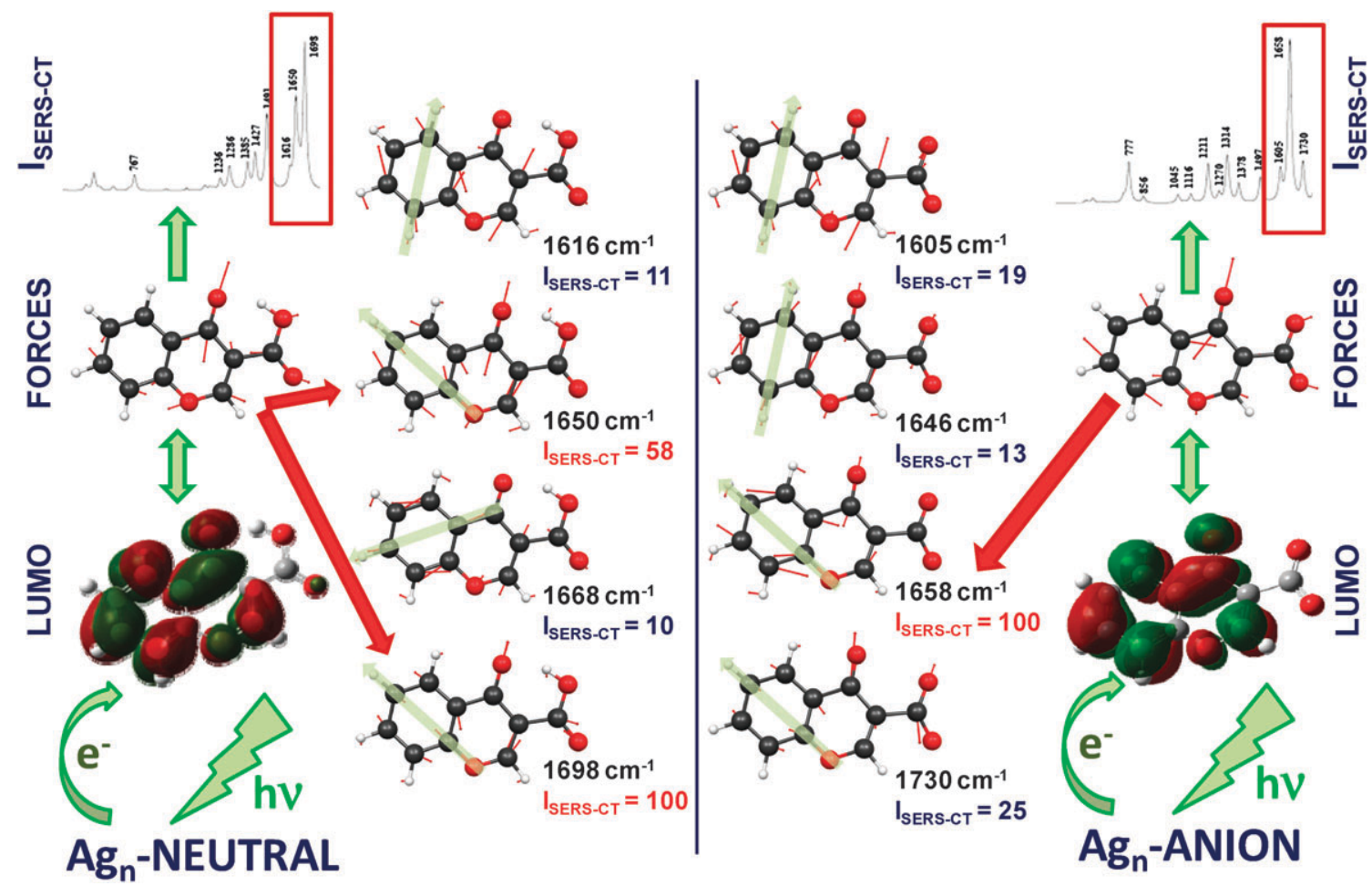

Fig. 3 Pictorial representation of the B3LYP/6-31G** calculated normal modes of the acid (neutral) and deprotonated (anion) chromone-3carboxylic acid (3CA) in the $1600-1700 \mathrm{~cm}^{-1}$ region. Correlation between the shapes of the respective LUMO's, the calculated forces at the Franck-Condon point of the excited CT states and the calculated intensities ( $\left.I_{\text {SERS-CT }}\right)$ of the SERS spectra under resonant CT conditions, for both chemical species. 


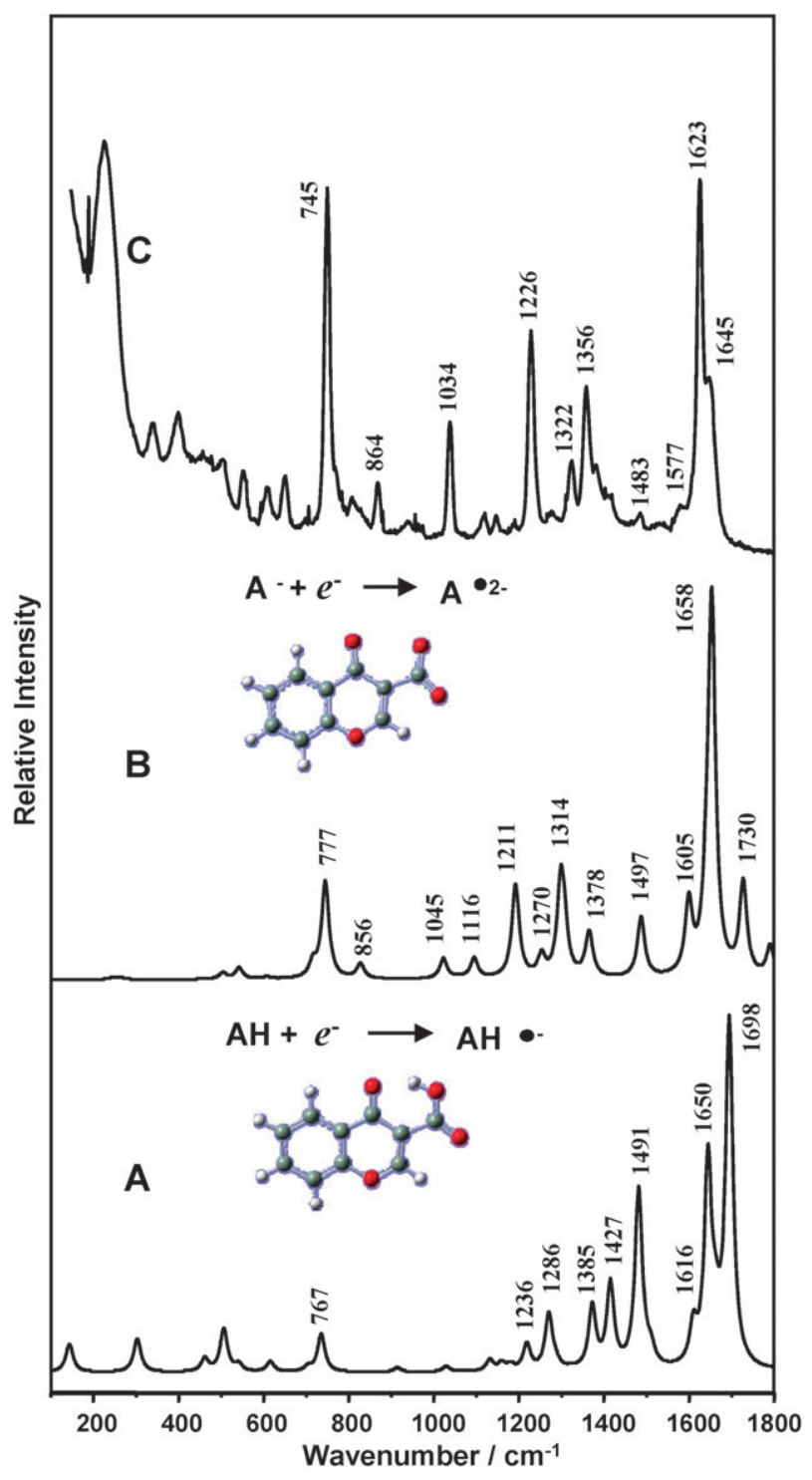

Fig. 4 Theoretical SERS-CT intensities for the neutral chromone-3carboxylic acid (3CA) (A) and the corresponding anion (B), and experimental SERS intensities (C).

for the modes calculated at 1605,1658 and $1730 \mathrm{~cm}^{-1}$ is much poorer, yielding $I_{\text {SERS-CT }}$ intensities of 19,13 and 25 , respectively. Moreover, the displacements of the $\mathrm{O}_{10} \mathrm{C}_{9} \mathrm{O}_{11}$ carboxylate atoms in the respective force vector, which are absent in the $1658 \mathrm{~cm}^{-1}$ mode, cause an enhancement of the 1314, 1211 and $777 \mathrm{~cm}^{-1}$ calculated fundamentals and account for the weak SERS activity evidenced by the bands at 1356, 1226 and $745 \mathrm{~cm}^{-1}$, respectively (Fig. 4).

When protonated $3 \mathrm{CA}$ is the adsorbate, a parallel analysis can be carried out from the results comprised in Fig. 3. The LUMO and the forces for both species, the anion and the neutral, look very similar, but for the latter the force vector cannot be easily correlated to one fundamental only. In fact, the SERS-CT enhancement for neutral 3CA is shared between the calculated modes at 1650 and $1698 \mathrm{~cm}^{-1}$, predicting relative intensities of 58 and 100, respectively. This produces a poorer correlation with the experimental SERS (Fig. 4) and discards the participation of protonated $3 \mathrm{CA}$ in the $\mathrm{CT}$ process.

Regarding the Raman and SERS wavenumbers, the observed shifts between both spectra point to an association of the 3CA molecule to the metal through the carboxylate group. For instance, the strong SERS band at $745 \mathrm{~cm}^{-1}$ is observed at $762 \mathrm{~cm}^{-1}$ in the aqueous solution Raman spectra, and corresponds to an in-plane vibration calculated at $777 \mathrm{~cm}^{-1}$ with a large contribution of the $\Delta\left(\mathrm{O}_{10} \mathrm{C}_{9} \mathrm{O}_{11}\right)$ internal coordinate. In a similar way, the $\nu_{\mathrm{s}}\left(\mathrm{O}_{10} \mathrm{C}_{9} \mathrm{O}_{11}\right)$ vibration is shifted from $1365 \mathrm{~cm}^{-1}$ in the Raman to $1356 \mathrm{~cm}^{-1}$ in SERS. It was verified that the B3LYP force field underestimates the wavenumber for this fundamental $\left(1314 \mathrm{~cm}^{-1}\right)$, due to the well known limitation of the theoretical force fields for predicting the coupling between the $\mathrm{CO}$ bonds of carboxylate groups. ${ }^{36,37}$ Similarly, the $\nu_{\text {as }}\left(\mathrm{O}_{10} \mathrm{C}_{9} \mathrm{O}_{11}\right)$ value is overestimated at $1730 \mathrm{~cm}^{-1}$.

\section{Conclusions}

The capability of 3CA to act as an electron scavenger is a very important property, closely related to its pharmacological activity as an antioxidant and potential chemopreventive agent, namely against cancer. In this concern, the surfaceenhanced Raman scattering (SERS) of 3CA adsorbed on silver sols has been analysed, in order to detect the presence of charge transfer (CT) processes. This resonant SERS-CT enhancement mechanism involves the photoinduced transfer of an electron from the metal to the adsorbate, which renders SERS a very useful spectroscopic technique for estimating electron affinities of chromones, flavones and related systems.

Apart from this specific goal, the present study aimed at a second objective, which was to determine the nature of the enhancement mechanism participating in the SERS which has been controversial for many years. Nowadays it is recognised that the electromagnetic (EM) mechanism is the main contribution, and that can be accompanied by resonant CT processes under particular conditions. For the 3-carboxylic chromone under study, theoretical SERS-CT intensities have been estimated for both the neutral $3 \mathrm{CA}$ and its deprotonated anion. This was performed using a methodology developed by our group that correlates the forces acting in the molecule when the transferred electron is located in the corresponding LUMO with the normal vibrational modes. The theoretical SERS-CT intensities calculated for the anionic species predict the selective enhancement of the band located at $1658 \mathrm{~cm}^{-1}$, in perfect agreement with the selective enhancement observed for the SERS feature at $1623 \mathrm{~cm}^{-1}$. This result supports the conclusion that the observed SERS is enhanced through a CT process, and confirms the capability of 3CA for accepting electrons. This is a quite relevant result, given that this molecule is already deprotonated when it adsorbs to the colloid, and therefore the SERS-CT mechanism implies the transient formation of the radical dianion. This work once again corroborates the relevance of the charge transfer mechanism in SERS for this type of polyphenolic conjugated systems and demonstrates the usefulness of the used methodology for detecting the relative contribution of this enhancement mechanism. In fact the 3CA molecule is a challenge for testing 
such methodology since it is a more complex system than the benzene-like derivatives studied to this date. ${ }^{13-15,27-33}$

\section{Acknowledgements}

This work was funded by the Spanish Ministerio de Ciencia e Innovación (Project Number CTQ2009-08549) and Junta de Andalucía (Project Number FQM-01895). N. F. L. Machado and C. Ruano kindly acknowledge FCT and MICINN for their PhD grants SFRH/BD/40235/2007 and BES 2007-17250

\section{References}

1 A. Foroumadi, A. Samzadeh-Kermani, S. Emami, G. Dehghan, M. Sorkhi, F. Arabsorkhi, M. R. Heidari, M. Abdollahi and A. Shafiee, Bioorg. Med. Chem. Lett., 2007, 17, 6764-6769.

2 H. Wei, M.-Z. Liu, Y. Li, Y. Tan and G.-F. Yang, Bioorg. Med. Chem., 2007, 15, 5191-5197.

3 Y. Li, H. Fang and W. Xu, Mini-Rev. Med. Chem., 2007, 7, 663-678.

4 M. Rode, R. C. Gupta, B. K. Karale and S. S. Rindhe, J. Heterocycl. Chem., 2008, 45, 1597-1602.

5 W. Huang, Y. Ding, Y. Miao, M.-Z. Liu and Y. Li, Eur. J. Med. Chem., 2009, 44, 3687-3696.

6 A. Gomes, O. Neuwirth, M. Freitas, D. Couto, D. Ribeiro, A. G. P. R. Figueiredo, A. M. S. Silva, R. S. G. R. Seixas and D. C. G. A. Pinto, Bioorg. Med. Chem., 2009, 17, 7218-7226.

7 M. Kuroda, S. Uchida, K. Watanabe and Y. Mimaki, Phytochemicals, 2009, 70, 288-293.

8 N. F. L. Machado and M. P. M. Marques, Curr. Bioact. Compd., 2010, 6, 76-89.

9 G. M. Williams, M. J. Iatropoulos and J. Whysner, Food Chem. Toxicol., 1999, 37, 1027-1038.

10 K. Roy and I. Mitra, Expert Opin. Drug Discovery, 2009, 4, $1157-1175$.

11 M. Moskovits, D. P. diLella and K. J. Maynard, Langmuir, 1988, 4, 67-76.

12 H. Metiu and P. Das, Annu. Rev. Phys. Chem., 1984, 35, 507-536.

13 S. P. Centeno, I. Lopez-Tocón, J. F. Arenas, J. Soto and J. C. Otero, J. Phys. Chem. B, 2006, 110, 14916-1922.

14 J. F. Arenas, J. Soto, I. Lopez-Tocón, D. J. Fernandez, J. C. Otero and J. J. Marcos, J. Chem. Phys., 2002, 116, 7207-7216.

15 I. López-Tocón, S. P. Centeno, J. L. Castro, M. R. López-Ramírez and J. C. Otero, Chem. Phys. Lett., 2003, 377, 111-118.

16 J. A. Creighton, C. G. Blatchford and M. G. Albrecht, J. Chem. Soc., Faraday Trans. 2, 1979, 75, 790-798.

17 J. L. Castro, M. R. López-Ramirez, I. López-Tocón and J. C. Otero, J. Colloid Interface Sci., 2003, 263, 357-363.

18 M. J. Frisch, G. W. Trucks, H. B. Schlegel, G. E. Scuseria, M. A. Robb, J. R. Cheeseman, J. A. Montgomery, Jr., T. Vreven, K. N. Kudin, J. C. Burant, J. M. Millam, S. S. Iyengar, J. Tomasi, V. Barone, B. Mennucci, M. Cossi,
G. Scalmani, N. Rega, G. A. Petersson, H. Nakatsuji, M. Hada, M. Ehara, K. Toyota, R. Fukuda, J. Hasegawa, M. Ishida, T. Nakajima, Y. Honda, O. Kitao, H. Nakai, M. Klene, X. Li, J. E. Knox, H. P. Hratchian, J. B. Cross, V. Bakken, C. Adamo, J. Jaramillo, R. Gomperts, R. E. Stratmann, O. Yazyev, A. J. Austin, R. Cammi, C. Pomelli, J. W. Ochterski, P. Y. Ayala, K. Morokuma, G. A. Voth, P. Salvador, J. J. Dannenberg, V. G. Zakrzewski, S. Dapprich, A. D. Daniels, M. C. Strain, O. Farkas, D. K. Malick, A. D. Rabuck, K. Raghavachari, J. B. Foresman, J. V. Ortiz, Q. Cui, A. G. Baboul, S. Clifford, J. Cioslowski, B. B. Stefanov, G. Liu, A. Liashenko, P. Piskorz, I. Komaromi, R. L. Martin, D. J. Fox, T. Keith, M. A. Al-Laham, C. Y. Peng, A. Nanayakkara, M. Challacombe, P. M. W. Gill, B. Johnson, W. Chen, M. W. Wong, C. Gonzalez and J. A. Pople, Gaussian 03, Revision D.01, Gaussian, Inc., Wallingford CT, 2004.

19 C. Lee, W. Yang and R. G. Parr, Phys. Rev. B: Condens. Matter, 1988, 37, 785-789.

20 B. Miehlich, A. Savin, H. Stoll and H. Preuss, Chem. Phys. Lett., 1989, 157, 200-206.

21 A. J. Becke, Phys. Rev. A: At., Mol., Opt. Phys., 1988, 38, 3098-3100.

22 A. J. Becke, J. Chem. Phys., 1993, 98, 5648-5652.

23 G. A. Petersson, A. Bennett, T. G. Tensfeldt, M. A. Al-Laham, W. A. Shirley and J. Mantzaris, J. Chem. Phys., 1988, 89, 2193-2218.

24 C. Peng, P. Y. Ayala, H. B. Schlegel and M. J. Frisch, J. Comput. Chem., 1996, 17, 49-56.

25 R. J. H. Clark and T. J. Dines, Angew. Chem., Int. Ed. Engl., 1986, 25, 131-158.

26 A. ten Wolde, H. J. C. Jacobs, F. W. Langkilde, K. Bajdor, R. Wilbrandt, F. Negri, F. Zerbetto and G. Orlandi, J. Phys. Chem., 1994, 98, 9437-9445.

27 J. F. Arenas, I. López-Tocón, J. C. Otero and J. L. Marcos, J. Chem. Phys., 1996, 100, 9254-9261.

28 J. F. Arenas, M. S. Woolley, I. López-Tocón, J. C. Otero and J. L. Marcos, J. Chem. Phys., 2000, 112, 7669-7683.

29 J. F. Arenas, S. P. Centeno, I. López-Tocón and J. C. Otero, Vib. Spectrosc., 2004, 35, 39-44.

30 J. F. Arenas, J. C. Otero, S. P. Centeno, I. López-Tocón and J. Soto, Surf. Sci., 2002, 511, 163-170.

31 M. Sardo, C. Ruano, J. L. Castro, I. López-Tocón, J. Soto, P. Ribeiro-Claro and J. C. Otero, Phys. Chem. Chem. Phys., 2009, 11, 7437-7443.

32 J. L. Castro, M. R. López-Ramírez, J. F. Arenas and J. C. Otero, Vib. Spectrosc., 2005, 39, 240-243.

33 J. L. Castro, J. F. Arenas, M. R. López-Ramírez, D. Peláez and J. C. Otero, J. Colloid Interface Sci., 2009, 332, 130-135.

34 E. B. Wilson Jr., Phys. Rev., 1934, 45, 706-711.

35 G. Varsányi, in Assignments for Vibrational Spectra of Seven Hundred Benzene Derivates, Akdémiai Kiadó,Budapest/Adam Hilger Ltd., London, 1974.

36 N. Biswas, S. Kapoor, H. S. Mahal and T. Mukherjee, Chem. Phys. Lett., 2007, 444, 338-345.

37 C. Jing and Y. Fang, Chem. Phys., 2007, 332, 27-32. 